\section{HISTOPATHOLOGY OF INTERSPINCTERIC RESECTION SPECIMENS OF LOW RECTAL CANCER: AN INSTITUTIONAL EXPERIENCE OF A SERIES OF 41 PATIENTS}

Mukta Ramadwar, Kedar Deodhar, Munita Bal, Avanish Sakalani, Reena Engineer, Supreeta Arya, Ashwin Desouza, Vikas Otswal

Tata Memorial Hospital, Mumbai, India

Background: Intersphincteric resection (ISR) for low rectal cancers is increasingly being offered in highly selected patients. It is often challenging with regard to the balance between tumour free margins and function of external sphincter.

Aim: We aim to review a series of 41 patients who have undergone ISR and examine histopathological parameters related to tumour including resection margins.

Methods: We reviewed clinical details, operative notes and detailed histopathology of 41 patients who underwent ISR in the year 2013-14.

Results: Age range of patients was $23-75$ years, median being 46 years. 34 of 41 patients received neo-adjuvant chemo-radiotherapy. Assessment of distal margin by frozen section was not performed in 13 of 41 patients, 2 of which revealed positive distal margin and circumferential margin (CRM) respectively and underwent abdominoperineal resection at a later date. Three specimens had a positive distal margin. $17 \%$ patients showed complete response to neo-adjuvant chemo-radiotherapy. $40 \%$ of patients had TRG score 3 . Two patients had tubulovillous adenoma, $17 \%$ had $\mathrm{T} 1$ adenocarcinoma, $41 \%$ had $\mathrm{T} 2$ adenocarcinoma and 35\% patients had T3 adenocarcinoma. Lymph nodal metastasis was seen in $31 \%$ of patients. CRM was involved in $12 \%$ of patients.

Conclusion: Intersphincteric resection for low rectal cancer is offered to a highly selected group of patients in our institute. Histopathology examination is crucial in assessment of distal mucosal margin intra-operatively. Intra-operative assessment of CRM by frozen section remains a limitation due to technical feasibility. A positive CRM at final histopathology examination would warrant a revision APR. In spite of neo-adjuvant therapy, the incidence of $\mathrm{T} 3$ tumours remained high.

\section{VALUE OF CHARACTERISTIC 'WEAK TO ABSENT' INI1/SMARCB1/BAF47 EXPRESSION IN DIAGNOSIS OF SYNOVIAL SARCOMAS}

Bharat Rekhi ${ }^{1}$, Ulrich Vogel ${ }^{2}$

${ }^{1}$ Department of Pathology, Tata Memorial Centre, Mumbai, India; and ${ }^{2}$ Institute of Pathology, University Hospital Tuebingen, EB-K University, Germany

Background: Lately, very few studies have shown diagnostic value of INI1/SMARCB1/BAF47 expression in synovial sarcomas (SSs).

Aims: To evaluate immunohistochemical (IHC) expression of INI1/SMARCB1 in SSs and in other tumours, including differential diagnoses of SSs.

Methods: Sixty-eight SSs, in the form of various specimens, including 26 cases confirmed with positive molecular cytogenetic results (SYT rearrangement), using Vysis SYT dual color break apart probe; along with 151 other tumours were tested for IHC expression of INI1.

Results: Forty monophasic spindle cell-type (58.8\%), 13 biphasic (19.1\%), 12 poorly differentiated (17.6\%) and 3 calcifying-type SSs $(4.4 \%)$ were positive for $\operatorname{EMA}(46 / 62,74.1 \%)$, AE1/AE3 (31/47, 65.9\%), CK 7 (20/31, 64.5\%), BCL2 (62/66, 93.9\%), MIC2 (cytoplasmic) (61/63, 96.8\%), TLE1 (diffuse) (29/31, 93.5\%), CK19 (14/24, 58.3\%), S100-P (1/13, 7.6\%) and CD34 (0/52). INI1 expression was 'weak to absent' in $60 / 68$ $(88.2 \%) \mathrm{SSs}$; in $1 / 3$ malignant ossifying myxoid tumours (MOFMTs) and in 3/10 (30\%) malignant peripheral nerve sheath tumours (MPNSTs) of various types, while 'completely absent' in 10/10 (100\%) epithelioid sarcomas (ESs), 4/4 (100\%) malignant rhabdoid tumours, single pediatric undifferentiated sarcoma, 5/19 (26.3\%) myoepithelial carcinomas and in 2/4 (50\%) epithelioid subtype of the MPNSTs. Remaining 104 tumours, including 12 Ewing sarcomas, 15 carcinomas, 8 solitary fibrous tumours (SFT), 7 extraskeletal myxoid chondrosarcomas and various other tumours retained INI1 expression.

Conclusions: A unique 'weak to absent' INI1 expression is highly sensitive $(88.2 \%)$ and specific $(97.3 \%)$ for a SS, irrespective of its various subtypes and biopsy specimens, especially in differentiating it from Ewing sarcoma, ES, SFT, carcinomas and some MPNSTs. A similar INI1 expression is also seen in few OFMTs. INI1 may be included as a useful marker in an optimal diagnostic panel of a SS comprising other markers such as EMA, AE1/AE3, BCL2, MIC2, TLE1 and CD34, especially in settings lacking molecular and/or cytogenetic analysis.

\section{CLINICOPATHOLOGICAL SPECTRUM OF A SERIES OF MERKEL CELL CARCINOMAS DIAGNOSED AT A TERTIARY CANCER REFERRAL CENTRE, INDIA}

Bharat Rekhi, Shubada V. Kane, Nirmala A. Jambhekar Department of Pathology, Tata Memorial Centre, Mumbai, India

Background: Merkel cell carcinoma (MCC) is a rare, primary cutaneous neuroendocrine carcinoma, characterized by an aggressive clinical course.

Aims: To present clinicopathological spectrum of a series of MCCs diagnosed at a tertiary cancer referral centre.

Methods: 16 MCCs, with immunohistochemical (IHC) staining results were analyzed after review.

Results: Sixteen primary MCCs occurred in 10 males and 6 females (M:F=1.6:1), between the ages 37 and 74 years (mean, 58.3, median, 58.6), commonly in lower extremities $(7,43.7 \%)$ and head and neck sites $(5,31.2 \%)$, followed by upper extremities $(3,18.7 \%)$ and abdominal wall (1). Tumour size varied from $0.5-9.9 \mathrm{~cm}$. Histopathologically, most tumours were composed of malignant round cells, mostly arranged diffusely with hyperchromatic nuclei, prominent apoptosis, mitoses and variable necrosis, including 'sudden' pleomorphism in some tumours. Variable features included co-existing Bowen's disease (2/16), along with squamous, glandular and rhabdomyoblastic de-differentiation (single tumor). Immunohistochemically, tumour cells were positive for at least a single epithelial marker 